\title{
A!
}

This is an electronic reprint of the original article.

This reprint may differ from the original in pagination and typographic detail.

Saarikoski, H.; Harju, A.; Puska, M.J.; Nieminen, R.M.

\section{Vortex Clusters in Quantum Dots}

Published in:

Physical Review Letters

DOI:

10.1103/PhysRevLett.93.116802

Published: 07/09/2004

Document Version

Publisher's PDF, also known as Version of record

Please cite the original version:

Saarikoski, H., Harju, A., Puska, M. J., \& Nieminen, R. M. (2004). Vortex Clusters in Quantum Dots. Physical Review Letters, 93(11), 1-4. [116802]. https://doi.org/10.1103/PhysRevLett.93.116802

This material is protected by copyright and other intellectual property rights, and duplication or sale of all or part of any of the repository collections is not permitted, except that material may be duplicated by you for your research use or educational purposes in electronic or print form. You must obtain permission for any other use. Electronic or print copies may not be offered, whether for sale or otherwise to anyone who is not an authorised user. 


\title{
Vortex Clusters in Quantum Dots
}

\author{
H. Saarikoski, A. Harju, M. J. Puska, and R. M. Nieminen \\ Laboratory of Physics, Helsinki University of Technology, P.O. Box 1100, FIN-02015 HUT, Finland
}

(Received 19 February 2004; published 7 September 2004)

\begin{abstract}
We study electronic structures of two-dimensional quantum dots in strong magnetic fields using mean-field density-functional theory and exact diagonalization. Our numerically accurate mean-field solutions show a reconstruction of the uniform-density electron droplet when the magnetic field flux quanta enter one by one the dot in stronger fields. These quanta correspond to repelling vortices forming polygonal clusters inside the dot. We find similar structures in the exact treatment of the problem by constructing a conditional operator for the analysis. We discuss important differences and limitations of the methods used.
\end{abstract}

DOI: $10.1103 /$ PhysRevLett.93.116802

PACS numbers: 73.21.La, 71.10.-w, 85.35.Be

Vortices appear in many physical systems from tornadoes and bathtub whirlpools [1] to type-II superconductors and rotating Bose-Einstein condensates [2]. In the fractional quantum Hall effect (FQHE) [3], the external magnetic field $B$ penetrates through the two-dimensional (2D) electron system at the vortex positions. Every vortex corresponds to a single magnetic field flux quantum. For the quantum Hall state of the filling factor $\nu=1$, a single vortex is on top of each electron. For stronger $B$, more vortices appear and, e.g., the Laughlin state of $\nu=1 / 3$ attaches three vortices on top of each electron. The vortices keep electrons farther apart, reducing the interaction energy and causing strong correlations between the electrons.

In this Letter, we report results of detailed numerical investigations of the electronic structure of 2D quantum dots (QDs) [4] in strong $B$. We use both a mean-field and an exact many-body approach. The $B$ values used are such that our QD states are related to the FQHE filling $1 \leq$ $\nu \leq 1 / 3$. We find both by the mean-field and the exact approach that vortices appear one by one inside QD as we strengthen $B$. The positions of these vortices are fixed in the mean-field solutions and are visible as zeros in the electron densities. The vortices form a polygonal cluster inside the QD. In the exact treatment the vortices are mobile. However, by constructing a conditional singleparticle wave function we are able to pinpoint them. Every electron binds one vortex, and there are also additional vortices that are not bound to any particular electron. These additional vortices form similar vortex clusters as found in the mean-field approach. Similar to the FQHE, the positions of the additional vortices are such that they reduce the interaction energy.

We model the 2D QD by an effective-mass Hamiltonian

$$
H=\left[\sum_{i=1}^{N} \frac{\left(-i \hbar \nabla_{i}+e \mathbf{A}\right)^{2}}{2 m^{*}}+V_{\mathrm{c}}\left(r_{i}\right)\right]+\frac{e^{2}}{4 \pi \epsilon} \sum_{i<j} \frac{1}{r_{i j}},
$$

where $N$ is the number of electrons in the dot, $\mathbf{A}$ is the vector potential of the perpendicular $B, m^{*}$ is the effective electron mass, and $\epsilon$ is the dielectric constant of the medium. We use a parabolic confinement $V_{\mathrm{c}}(r)=$ $\frac{1}{2} m^{*} \omega_{0}^{2} r^{2}$, and the material parameters of GaAs, $m^{*} / m_{e}=$ 0.067 , and $\epsilon / \epsilon_{0}=12.4$. We assume the Zeeman effect to be strong enough to spin polarize our system in the $B$ range considered. We solve the model using both the mean-field density-functional theory (DFT) and the exact diagonalization (ED). We use two variants of DFT, namely, the spin-DFT (SDFT) and the current-spinDFT (CSDFT) [5]. The DFT solutions are found on real-space grids without symmetry restrictions [6]. Special effort is laid on the numerical accuracy, and the convergence is tested with large grids up to the size of $256 \times 256$ grid points. The exchange-correlation effects are taken into account using local approximations [7]. In CSDFT, the effect of currents is also included in the exchange-correlation functionals, again in a local approximation. CSDFT is computationally more demanding than SDFT. As both schemes give qualitatively similar results, we have mainly used SDFT. Our ED calculations use wave functions restricted to the lowest Landau level (LLL) [8-10].

The starting point for our study is a $B$ value where the maximum-density-droplet (MDD) state is formed [11]. This is a finite-size precursor of the $\nu=1$ quantum Hall state [3]. This state can be found in various QD geometries, and it is characterized by a rather flat and compact electron density [12]. In our case, MDD is formed by the LLL orbitals with angular momentum $l=0,1, \ldots, N-$ 1 , and the total angular momenta $L$ equals $N(N-1) / 2$. In MDD, one vortex is bound to each electron to give the correct fermion nature in the LLL wave function.

Our study focuses on large $B$ values beyond the MDD region, where ED shows the ground states to occur only at certain "magic" $L$ values, and $L$ exhibits a stepwise structure as a function of $B[10,13,14]$. We show that the ground states in this region can be characterized by an increasing number of additional vortices entering the QD 
and forming vortex clusters. We first analyze the postMDD region of a six-electron QD. Setting the confinement strength to $\hbar \omega_{0}=5 \mathrm{meV}$, SDFT predicts the MDD formation at $B \approx 5 \mathrm{~T}$, and the state is a ground state up to $B \approx 8 \mathrm{~T}$ [6]. Figure 1 shows the SDFT result for $L$ as a function of $B$. The ED ground-state $L$ values, which agree with the previous calculations [14], are given by the dashed lines. The values $L=15+6 n$, where $n$ is an integer, reflect the sixfold rotational symmetry, whereas values $L=15+5 n$ correspond to the fivefold rotational symmetry [10]. The SDFT results show plateaus with a small slope just at the magic $L$ values. A possible exception is the $L=39$ magic momentum which is not clearly visible in the SDFT results. One should note that the unrestricted Hartree-Fock approximation has been shown to follow the trend in angular momentum but not to reproduce the staircase structure [15]. As shown by densities in Figs. 2(a)-2(c), the plateaus are characterized by vortex holes, i.e., rotating currents with zero electron density at the center. The number of vortices increases by one between plateaus of nearly constant $L$. There are vortices also farther away from the dot center, where the electron density is a tiny fraction of the maximum density.

To compare our ED results with the DFT ones, we have constructed an operator that efficiently shows the positions of the vortices in the system. We first find the most probable electron positions $\left\{\mathbf{r}_{i}^{*}\right\}_{i=1}^{N}$ by maximizing the density $|\Psi|^{2}$. One of the electrons is then moved to a new position $\mathbf{r}$, and a conditional single-particle wave function is evaluated as

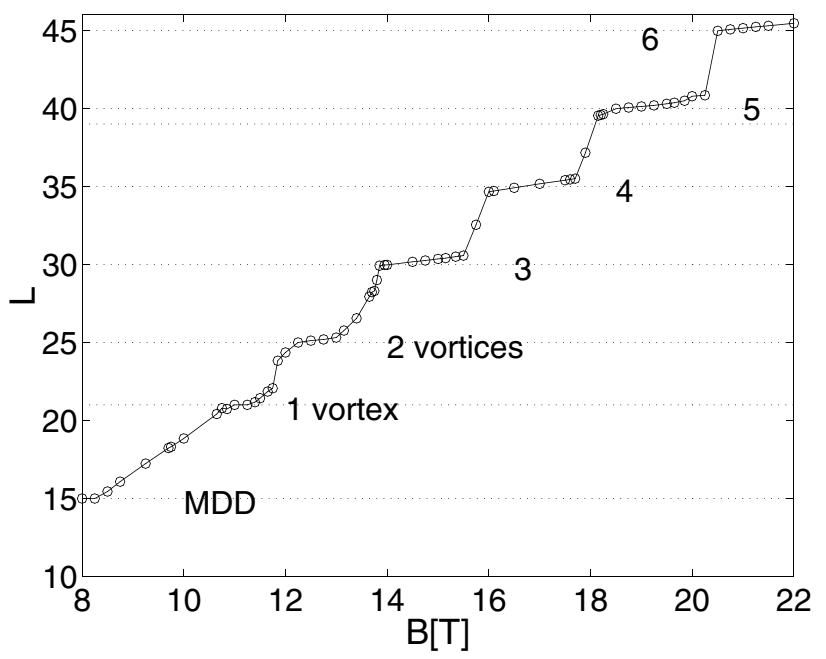

FIG. 1. Angular-momentum $L$ of the six-electron QD from SDFT (open circles). The plateaus are characterized by vortex holes in the electron density. The number next to a plateau gives the number of vortices inside QD. The horizontal lines correspond to ground-state $L$ values from ED.

$$
\psi_{\mathrm{c}}(\mathbf{r})=\frac{\Psi\left(\mathbf{r}, \mathbf{r}_{2}^{*}, \ldots, \mathbf{r}_{N}^{*}\right)}{\Psi\left(\mathbf{r}_{1}^{*}, \mathbf{r}_{2}^{*}, \ldots, \mathbf{r}_{N}^{*}\right)}
$$

The change in the phase can be obtained from the angle $\theta$ of the wave function $\psi_{\mathrm{c}}(\mathbf{r})=\left|\psi_{\mathrm{c}}(\mathbf{r})\right| \exp (i \theta(\mathbf{r}))$. We have plotted $\psi_{\mathrm{c}}$ in Figs. 2(d)-2(f). The electron density is shown using contours, and the phase is shown using a gray scale to indicate the angle. The vortices show up as zeros in $\psi_{\mathrm{c}}$. A rotation around one vortex changes the phase by $2 \pi$. One can see that the number of additional vortices in the inner part of the QD agrees with our DFT results. There are also vortices outside the ring of fixed electrons, as in the CSDFT case. One should note that in the $L=45$ solution of Fig. 2(f), the total number of vortices close to each fixed electron is three, as in the Laughlin wave function for the $\nu=1 / 3$ quantum Hall state [3]. Unlike in the Laughlin state, there is a repulsion between the vortices, forcing two of them to stay on the opposite sides of the fixed electron. However, the overlap between the Laughlin and the exact states is high, 0.98 .

The electron densities in Figs. 2(d)-2(f) also show the Wigner-molecule formation [16]. In Fig. 2(d) the conditional density is still well spread over the whole QD (consider, e.g., the third contour from the top, corresponding to the density $e^{-5} \approx 0.007$ ), whereas in Fig. 2(f) the density is strongly peaked around the most probable position of the probe electron. One should note that the most probable electron positions approach the classical positions in the Wigner-molecule limit. For the Coulomb interaction, the classical configuration of six point charges is a pentagon with one electron in the center. This result is in accord also with the CSDFT solution for the six-vortex case which shows an electron in the
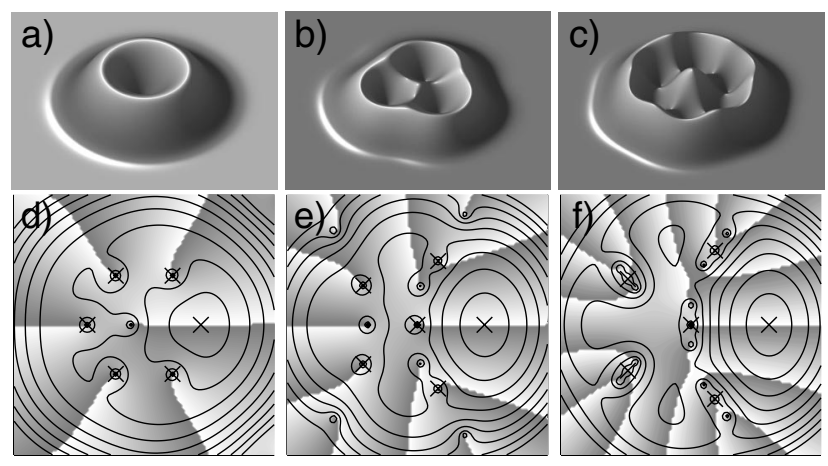

FIG. 2. Upper panel: Electron densities of the six-electron QD calculated with CSDFT for single-vortex (a), three-vortex (b), and six-vortex (c) configurations. Lower panel: Most probable electron positions $\left\{\mathbf{r}_{i}^{*}\right\}_{i=1}^{N}$ (crosses) and conditional electron densities (contours) and phases (gray scale) from ED for the cases of the upper panel: $L=21$ (d), $L=30$ (e), and $L=45$ (f). We probe with the rightmost electron. The densities are on a logarithmic scale (values $e^{-i}, i=1,3, \ldots, 17$ ), and the phase changes from $\pi$ to $-\pi$ on the lines where shadowing changes from the darkest gray to white. 
middle and a five-electron ring. This is also the most probable electron configuration in most ED ground states, the one in Fig. 2(d) being one of the exceptions having the hexagonal configuration. In many ground states, symmetry eliminates one of the two possible configurations. For the $L=45$ case of Fig. 2(f), the hexagonal configuration has also a high probability, namely, of $77 \%$ of the pentagonal one, showing that the electrons still make multiparticle exchanges in this ground state [16].

From the computational point of view, constructing the conditional wave function $\psi_{\mathrm{c}}$ is a demanding task. The basic reason for this is that it does not contain any integrations of coordinates, which leaves $\psi_{\mathrm{c}}$ a true $N$-particle operator. Thus one is forced to actually construct the Slater determinants in the ED wave function expansion. For efficient evaluation of the determinants, we have first decomposed the matrices to triangular ones. The computational cost in constructing $\psi_{\mathrm{c}}$ for the $L=45$ case with a grid size of $100 \times 100$ for $\mathbf{r}$ is 20 times more than that of a mere diagonalization. For this reason, previous ED works have mainly concentrated on operators that can be written using one-particle and twoparticle operators [10].

As the Hamiltonian of Eq. (1) is rotationally symmetric, the particle density should also have this property. Calculations, however, show that the particle density from DFT is not necessarily symmetric. Vortex solutions clearly break the rotational symmetry of the particle density in the case of more than one vortex. Other solutions with broken rotational symmetry are, e.g., spin-density-wave and charge-density-wave structures $[17,18]$. The analysis and interpretation of these solutions are highly nontrivial. Symmetry-breaking may result from an unphysical mixture of states that do not mix in an exact treatment $[19,20]$. However, for the vortex case, there are strong topological reasons behind the broken symmetry in DFT. Namely, if we suppose that there are vortices in the true many-body solution of the system, this directly forces the vortices to localize. This follows from considering the Kohn-Sham (KS) equations close to a vortex. The kinetic energy diverges if the electron density of the KS orbit does not vanish at the vortex position. As a
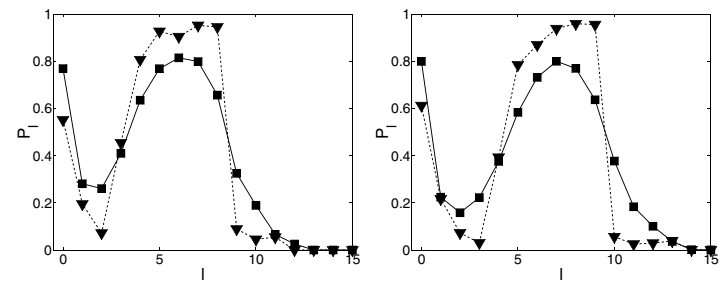

FIG. 3. Occupation of angular-momentum eigenstates for solutions with three (left) and four (right) vortices. Triangles mark the CSDFT and squares the ED results, respectively. The minima at the low $l$ values show the formation of vortices in the QD. mean-field theory, DFT misses relative coordinates of the electrons, and thus the vortices must localize in space. In the true many-body solution of the system, however, the vortices move as the electron coordinates are changed. Thus the vortices behave as quantum-mechanical particles in the exact treatment, showing quantum-mechanical zero-point motion, but the mean-field approximation forces them to behave as classical particles.

The CSDFT and ED solutions are analyzed further by calculating the occupation of the angular-momentum eigenstates, i.e., the projections on the Fock-Darwin orbitals. For the MDD, all angular momenta $l \leq N-1$ have occupancy one and the others are zero. The singlevortex state of DFT has the $l=0$ orbital unoccupied and the following $l=1, \ldots, 6$ orbitals occupied. Figure 3 shows the occupations for three- and four-vortex solutions ( $L=30$ and 35, respectively). The missing occupation at low $l$ increases with the number of vortices. Thus vortices appear as holes in the MDD. As the magnetic field squeezes the QD, it becomes at some points favorable to extend the system by creating an additional vortex hole into the system [8]. From the occupation in Fig. 3 one can see that the DFT vortices are more localized to certain angular-momentum values than the ED ones. The SDFT occupations differ more from the ED results than the CSDFT ones.

We have performed SDFT calculations also for a 24electron QD, with the confinement strength changed to $\hbar \omega_{0}=1.8940 \mathrm{meV}$. The calculations predict the formation of clusters of vortices in high magnetic fields. Figure 4 shows the electron density at $5 \mathrm{~T}(L \approx 491)$. The currents in our DFT solutions circulate counterclockwise on the edge of the QD, whereas the circulation is clockwise around the vortices. This behavior is consistent with the classical picture of a conducting ring where the inner circulation of electrons reverses the current near the center hole [21]. Since the vortex holes behave as classical repelling particles in DFT, the vortex clusters inside the QD are usually similar to the configurations of classical point charges confined by a parabolic external potential [22]. For instance, the solution at $5 \mathrm{~T}$ contains 14 vortices arranged in the $(4,10)$ configuration, i.e., four vortices in the middle and ten in the second shell, which is the same

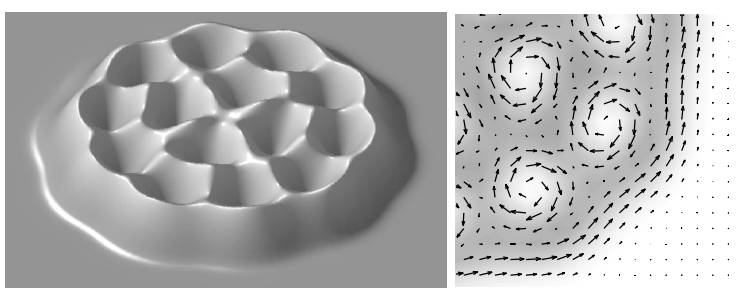

FIG. 4. Left: SDFT electron density of a 24-electron QD at 5 T showing 14 vortices. Right: Electron density (gray scale, the white regions have low electron density and correspond to vortices) and currents (arrows) on the edge of the QD. 
as the ground-state configuration for 14 classical particles. The positions of vortices are not independent of the electron degrees of freedom, and therefore the vortex configurations do not necessarily match those of the classical case. This can be seen in Fig. 2(c) where the vortex cluster is hexagonal in contrast to the $(1,5)$ configuration for the classical six-particle system.

In Bose-Einstein condensates, there is a repulsive interaction between the atoms, and the many-body wave function for a rotating condensate can be constructed similar to that for a $2 \mathrm{D}$ electron gas in a magnetic field [23]. This means that the two systems are analogous and explains why the mean-field solution for the rotating Bose-Einstein system also shows vortex clusters [2].

The DFT solutions between the plateaus show chargedensity-wave (CDW) solutions with a fractional $L$. In the first CDW domain $L$ increases linearly with $B$ from 15 to 21 (see Fig. 1). The particle density in this region shows six charge maxima in the form of a hexagon and with currents flowing counterclockwise around them. The radius of each charge lump is of the order of the magnetic interaction length $\ell_{B}=\sqrt{\hbar / e B}$. These solutions are reminiscent of the localized electron states found by Reimann et al. just above the MDD region [18]. Reimann et al. do not show results for the higher $B$ values where vortex solutions appear in our calculations. We want to underline that finding the vortex solutions requires high numerical accuracy. Our real-space scheme is superior to the planewave expansion of wave functions used by Reimann et al., especially in describing the vanishing electron density at the vortex core.

We have analyzed the angular-momentum occupations of the first CDW region between 15 and 21. The results show that these states are combinations of the $L=15$ and 21 states. A mixture of these two states results in a CDW with six peaks in the form of a hexagon. This result is in disaccord with the (excited state) ED solutions between $L=15$ and 21 , which show a vortex hole moving from the outer edge toward the center [9]. The DFT mixing of eigenstates belonging to different angular-momentum eigenvalues can be thought to be unphysical and resulting from limitations of DFT. These limitations can be seen also in the mixing of total spin states in SDFT [20]. Our analysis of the symmetry-breaking solutions shows that DFT, which is an indispensable tool for large systems, may reveal proper correlations between the electrons via these solutions. But the correct interpretation of the results requires careful analysis completed by, e.g., the ED method.

To conclude, we have found vortex clusters as meanfield solutions of the two-dimensional quantum dots in strong magnetic fields. The external magnetic field penetrates through the dot at the position of the vortices, inducing electron currents that circulate around them.
The exact treatment of the problem shows similar features in the conditional electron wave function. The mean-field approach has been shown to lack the quantum-mechanical nature of the vortices, leading to a vortex localization and a broken rotational symmetry for the cases with more than one additional vortex. Apart from these facts, DFT results have been shown to accurately describe the electronic structure of this challenging system, enabling one to study system sizes beyond reach of the more exact treatments. We hope that this theoretical prediction will inspire further work for actual experimental evidence of vortex formation in quantum dots. This could presumably be seen indirectly in the magnetization measurements of quantum dots. A more direct observation could be made if the vortices get localized due to a lower symmetry of the system, e.g., in quantum dots distorted by impurities. The stability and structure of vortices in nonsymmetric potentials is, however, a nontrivial problem, and further theoretical work is needed.

We thank S. M. Reimann and E. Räsänen for fruitful discussions. This work has been supported by Academy of Finland through the Centre of Excellence Program (2000-2005).

[1] A. Andersen et al., Phys. Rev. Lett. 91, 104502 (2003).

[2] D. A. Butts and D. S. Rokhsar, Nature (London) 397, 327 (1999).

[3] T. Chakraborty and P. Pietiläinen, The Quantum Hall Effects: Fractional and Integral (Springer, Berlin, 1995).

[4] For a recent review, see S. M. Reimann and M. Manninen, Rev. Mod. Phys. 74, 1283 (2002).

[5] G. Vignale and M. Rasolt, Phys. Rev. B 37, 10685 (1988).

[6] H. Saarikoski et al., Eur. Phys. J. B 26, 241 (2002).

[7] C. Attaccalite et al., Phys. Rev. Lett. 88, 256601 (2002).

[8] S.-R. Eric Yang and A. H. MacDonald, Phys. Rev. B 66, 041304(R) (2002).

[9] J. H. Oaknin et al., Phys. Rev. Lett. 74, 5120 (1995).

[10] T. Seki et al., J. Phys. Soc. Jpn. 65, 3945 (1996).

[11] A. H. MacDonald et al., Aust. J. Phys. 46, 345 (1993).

[12] E. Räsänen et al., Phys. Rev. B 69, 165309 (2004).

[13] P. A. Maksym and T. Chakraborty, Phys. Rev. Lett. 65, 108 (1992); P. A. Maksym, Phys. Rev. B 53, 10871 (1996).

[14] A. Wójs and P. Hawrylak, Phys. Rev. B 56, 13227 (1997).

[15] B. Szafran et al., Eur. Phys. J. D 28, 373 (2004).

[16] A. Harju et al., Phys. Rev. B 65, 075309 (2002).

[17] M. Koskinen et al., Phys. Rev. Lett. 79, 1389 (1997).

[18] S. M. Reimann et al., Phys. Rev. Lett. 83, 3270 (1999).

[19] K. Hirose and N. Wingreen, Phys. Rev. B 59, 4604 (1999).

[20] A. Harju et al., Phys. Rev. B 69, 153101 (2004).

[21] Craig S. Lent, Phys. Rev. B 43, 4179 (1991).

[22] V. M. Bedanov and F. M. Peeters, Phys. Rev. B 49, 2667 (1994).

[23] M. Manninen et al., Phys. Rev. B 64, 245322 (2001). 